Editorial

\title{
Editorial for the Special Issue on Graph-based representations in pattern recognition
}

Graph-based representations have been used with considerable success in computer vision and pattern recognition for over 40 years for their representational power and flexibility. Indeed, such representations arise naturally in many low-level and highlevel vision tasks and are in general central to several pattern matching and analysis approaches. However, despite their large use, structural representations pose unique problems in machine learning, due to their non-vectorial nature.

The goal of this special issue was to publish high-quality papers that provide a clear picture of the state-of-the-art of the use of graphs as a representational tool in computer vision and pattern recognition, and was meant to disseminate the best and most representative papers presented at the Eighth IAPR TC-15 International Workshop on Graph-based representation (GbR 2011). The guest editors selected 11 papers from 34 candidates and had each reviewed at least by two reviewers, with several being reviewed by three. The papers describe a diverse set of methods and applications.

The first paper presented in this special issue "Graph Characterizations From Von Neumann Entropy" by Han et al. analyzes the complexity of structural representations by studying the entropy of the spectra of the graph Laplacians.

"Invariants of Distance $k$-Graphs for Graph Embedding" by Czech presents new graph features based on the degrees of distance $k$-graphs, axillary structures representing extended neighborhoods, while in "Feature selection on node statistics based embedding of graphs", Gibert et al. address the selection of feature to represent structural information using different feature selection algorithms, such as PCA and ranking, based on the node statistics.

Zhang and Hancock, in "Hypergraph based Information-theoretic Feature Selection" addresses the problem of feature selection based on high-order feature dependencies with respect to the class labels. These dependencies are captured by means of an information-theoretic criterion referred to as multidimensional interaction information, and are abstracted in terms of a hypergraph. The most informative features are finally sought using a hypergraph clustering approach.

Based on recent results generalizing the central limit theorem on graph orbitfolds, Jain presents a method to infer an approximation of the "mean" and "standard deviation" of a probability distribution that represents graphs in "Maximum Likelihood Method for Parameter Estimation of Bell-Shaped Functions on Graphs."
Weber et al., in "Faster Subgraph Isomorphism Detection by Well-Founded Total Order Indexing" improve a graph indexing algorithm adopting a total order on the entries in the decision tree thus reducing the number of permutations to be stored in the decision tree, rendering it usable for larger graphs.

In "From Maximum Common Submaps to Edit Distances of Generalized Maps" Combier et al. present a distance measure for comparing generalized combinatorial maps by means of the size of a largest common submap. This allows also to import the concept of edit distance to maps.

"Two New Graphs Kernels in Chemoinformatics" by Gaüzère et al. introduce two graph kernels to gauge the similarity of molecular structures. The first is based on the edit distance between the structures, while the second is based on the decomposition of a graph into a set of distinct substructures called treelets.

Jiang et al. in "Structural Performance Evaluation of Curvilinear Structure Detection Algorithms with Application to Retinal Vessel Segmentation" address the problem of evaluating the segmentation of curvilinear structures, motivated by retinal image blood vessels. They find that the standard practice of performing a pixel-to-pixel comparison against a hand-labeled ground truth has some issues and propose to match a thinned segmentation result against a thinned ground truth.

Finally, in "A Graph-Based Technique for Semi-supervised Segmentation of 3D Surfaces" Bergamasco et al. propose a semi-supervised procedure to efficiently segment large-scale 3D meshed surfaces. The approach propagates part labels over the dual graph of the mesh in a way consistent with curvature variations.

The variety and high quality of the submitted papers demonstrate that the topics covered by the special issue are of great interest to the pattern recognition community. We hope that this collection of papers will provide both a timely and interesting sample of research in the field of graph-based representations.

Andrea Torsello Università Ca' Foscari Venezia, Italy Xiaoyi Jiang University of Münster, Germany Miquel Ferrer Universitat Autònoma de Barcelona, Spain 